\title{
Modelling local bending stiffness based on fibre orientation in sawn timber
}

\author{
Min $\mathrm{Hu}^{1}$ (1) $\cdot$ Anders Olsson ${ }^{1} \cdot$ Marie Johansson ${ }^{1} \cdot$ Jan Oscarsson $^{1}$
}

Received: 7 November 2017 / Published online: 7 September 2018

(c) The Author(s) 2018

\begin{abstract}
Strength of structural timber depends to a high degree on the occurrence of knots and on the local fibre deviation around such defects. Knowledge of local fibre orientation, obtained by laser scanning, has been utilized in a previously developed machine strength grading method, but rather crude assumptions regarding the fibre orientation in the interior of boards and a mechanical model that does not capture the full compliance of knotty sections were adopted. The purpose of the present study was to suggest and verify a model with which local bending stiffness can be predicted with high accuracy. This study included development of a model of fibre orientation in the interior of boards, and application of a three-dimensional finite element model that is able to capture the compliance of the board. Verification included bending of boards in the laboratory and application of digital image correlation to obtain strain fields comparable to those obtained by finite element simulation. Results presented comprise strain fields of boards subjected to bending and calculated bending stiffness profiles along boards. Comparisons of results indicated that the model suggested here was sufficient to capture the variation of local bending stiffness along boards with very high accuracy.
\end{abstract}

\section{Introduction}

\subsection{Background}

Development of accurate machine strength grading methods requires knowledge of the mechanical properties of timber, which depend on clear wood properties and influence of defects. Clear wood exhibits orthotropic properties. The material is much stronger and stiffer along fibres than in the transverse directions. Thus, strength and stiffness in clear wood are dependent on the angle between fibre and load directions, and such a relationship has already been described mathematically by Hankinson (1921). Knots, and the substantial local fibre deviation related to knots, are regarded the most important types of defect that considerably reduce the strength of structural timber (e.g. Johansson

Electronic supplementary material The online version of this article (https://doi.org/10.1007/s00107-018-1348-2) contains supplementary material, which is available to authorized users.

$\mathrm{Min} \mathrm{Hu}$

min.hu@lnu.se

1 Department of Building Technology, Linnaeus University, Växjö, Sweden et al. 1998; Johansson 2003). Therefore, gaining knowledge of local fibre orientation and using such information to develop models for the mechanical properties of individual boards are in focus of the current research with the longterm aim to develop grading methods more accurately than those available today.

\subsection{Models for fibre orientation in the vicinity of knots}

Attempts to create models that describe the fibre orientation close to knots have been made since the 1970s. Goodman and Bodig (1978) suggested a so-called flow-grain analogy model based on the visual similarity between the grain pattern in close-to-knot areas within a growth layer of a tree and the streamline pattern of laminar fluid flowing around an elliptically shaped obstacle. A growth layer is herein defined as a xylem cell layer that is developed at a certain point of time in the cambium area of the tree. Hence, stream functions were used to describe the fibre path around knots in the longitudinal-tangential plane of the wood material. Cramer and Goodman (1983) adopted the flow-grain analogy model suggested by Goodman and Bodig (1978) and applied the resulting fibre orientation in two-dimensional (2D) finite element (FE) models of two wood specimens each containing 
a single knot. These models were used for analysis of local stresses and strains of loaded members. The 2D flow-grain analogy was adopted and further developed by Foley (2001, 2003) into a three-dimensional (3D) model with which a complete set of local material directions can be determined at an arbitrary position. In Foley's model, the fibre angle projected onto the longitudinal-tangential plane of a growth layer is modelled by the flow-grain analogy, while the fibre orientation in the longitudinal-radial plane is determined on the basis of mathematical functions describing the observed geometry of growth layers in the vicinity of knots. Based on work by Hackspiel (2010), Lukacevic and Füssl (2014) presented an FE model of sawn timber where Foley's model was employed to establish fibre orientation in 3D in the surroundings of knots. The model was used to estimate stiffness and strength properties of individual boards, and subjected to experimental verifications. Knowledge of the size, position and shape of knots within a board is a prerequisite for application of Foley's model. In the work presented by Lukacevic and Füssl (2014), knot positions and knot geometries were detected by digital image processing of images of the board surfaces. Further attempts and algorithms to model knots in 3D within boards, based on the detection of knots on wood surfaces, have been presented by Briggert et al. (2016a) and Kandler et al. (2016). The results obtained were in both cases quite accurate regarding size and position of knots but not regarding the precise shape of the knots. Briggert et al. (2016b) showed that even a small deviance between the assumed and the real shape/curvature of the knot causes large deviation between the fibre orientation calculated using Foley's model and the corresponding fibre orientation observed on wood surfaces. Hence, the models of 3D knot geometry presented so far, based on information obtained by investigations of wood surfaces, do not provide a basis for very accurate determination of the fibre path around knots.

Another starting point for describing the fibre path around knots has been a principal stress based logic put forward by Mattheck (1997). The basic idea is that fibres around a knot are orientated in the directions of principal stresses due to natural loads during the growth of the tree. Based on this assumption, an initial stress analysis using an isotropic material model was carried out to determine directions of major principal stresses on a local level. Then, an orthotropic material model, where fibres at a local position are supposed to be oriented in the direction of the major principal stress previously determined at the same position, was introduced. Next, an iterative process based on the orthotropic material model was carried out. For each iteration, new directions of major principal stresses were determined and the iterative process was terminated when the direction of principal stresses no longer changed significantly between two successive iterations. Lang and Kaliske (2013) discussed and compared the flow-grain analogy and the principal stress based model and showed that the two models provide similar results regarding the fibre orientation around knots. However, they did not present any data from laboratory investigations to confirm the accuracy of calculated fibre orientation. Hu et al. (2018) presented results of the 3D fibre orientation around a knot in sawn timber of Norway spruce based on a close laboratory investigation.

\subsection{Laser scanning and the tracheid effect}

Matthews and Beech (1976) patented a method based on directing a spot of light to a timber surface to recognize knots and other defects. This was done by analysing the scattering of light on the surface. Although the purpose expressed in the patent was the detection of defects, rather than the detection of fibre orientation, they noted that "light travelling along grain is attenuated much less than light travelling across the grain" and thus they observed what is known as the tracheid effect. This means that a light spot (that would have been circular in shape on a surface of isotropic material) on the wood surface becomes elliptical in shape and that the major axis of the elliptically shaped light spot indicates the fibre orientation in the plane of the surface. Many researchers have contributed to further development and to enable practical utilization of the method (e.g. Seltman 1992; Soest et al. 1993; Nystörm 1999, 2003). Nowadays laser scanning using tracheid effect is common in industry scanners for the detection of fibre orientation. Figure 1a shows a surface image of a piece of wood including a knot, Fig. 1b spread of laser light on the wood surface, and Fig. 1c the identified in-plane fibre orientation on the wood surface.

The tracheid effect also gives some indication of the outof-plane fibre orientation, i.e. of the so-called diving angle (Simonaho et al. 2004), and this has sometimes been utilized in applied research, for example Briggert et al. (2016a) and $\mathrm{Hu}$ et al. (2016). However, the diving angles determined using the tracheid effect suffer from major uncertainties, which has been shown by Briggert et al. (2016b).

\subsection{Machine strength grading based on data of fibre orientation}

Knowledge of fibre orientation, which should enable accurate predictions of strength, has been utilized by researchers that have suggested new methods for grading purposes (e.g. Hackspiel 2010; Lukacevic and Füssl 2014; Viguier et al. 2017). However, machine strength grading that utilizes such knowledge has not until recently been available on the market. Olsson et al. (2013) presented a new grading method that utilizes data of fibre orientation on wood surfaces obtained from laser scanning. This method relies 


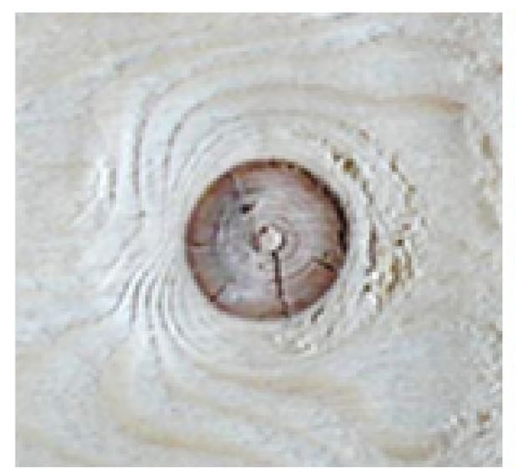

(a)

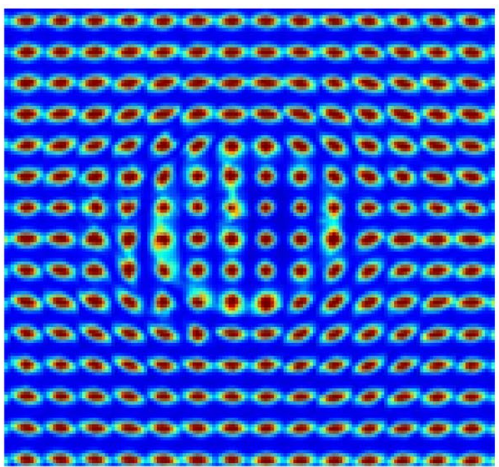

(b)

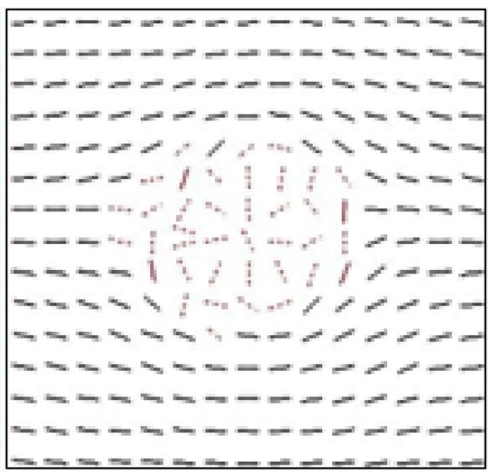

(c)

Fig. 1 a Wood surface including a knot, b spread of dot laser light on the wood surface, and $\mathbf{c}$ identified in-plane fibre orientation on the wood surface (images originate from Petersson 2010)

on a concept that can be divided and expressed in two parts, as follows:

1. Accurate prediction of strength can be based on the knowledge of local edgewise bending stiffness, i.e. on the knowledge of local bending MOE in different positions along a board,

2. It is possible to establish an accurate bending MOE profile for a board, i.e. to obtain knowledge of the local bending MOE in every position along it, on the basis of the knowledge of fibre orientation obtained by tracheid effect scanning, in combination with knowledge of axial dynamic MOE obtained by dynamic excitation and knowledge of the weight of the board.

Regarding the first part, many researchers in the past have expressed similar ideas, as shown by Oscarsson et al. (2014). The grading method suggested by Olsson et al. (2013) enables prediction of bending strength for Norway spruce that is more accurate than what other approved methods do. On a sample consisting of more than 900 boards of Norway spruce [Picea abies (L.) H. Karst.] the coefficient of determination $\left(R^{2}\right)$ between the suggested indicating property (IP) and the edgewise bending strength was 0.69 , whereas the $R^{2}$ value obtained for axial dynamic MOE vs. bending strength was only 0.53 (Olsson and Oscarsson 2017), confirming that the concept is useful. However, regarding the second part of the concept as it is expressed above, no thorough examination has actually been presented so far. It is also quite clear that assumptions made when utilizing knowledge of fibre orientation on surfaces and of axial dynamic MOE may be crucial for the accuracy of the established bending MOE profile. To enable a discussion on modelling that aims at the calculation of accurate local bending MOEs, a summary of the implementation made, divided in steps (a-e) is: (a) The in-plane fibre orientation, locally on face and edge surfaces of the board, was known from tracheid effect scanning.

(b) It was assumed that the in-plane fibre orientation, represented by an angle, $\varphi$, detected locally on a board surface, is representative for a small area (a few square millimetres, depending on the scanning resolution) of the surface. The $\varphi$ value on such an area is also valid to a certain depth into the board, i.e. the angle $\varphi$ exhibited in Fig. 2a was assumed valid for a certain small volume of the board.

(c) Values of nine independent material parameters of the wood material (MOEs, shear moduli and Poisson's ratios), were adopted. On the basis of these, and of each local fibre angle, $\varphi$, the corresponding local MOE, $E_{\mathrm{x}}(x, y, z)$, valid in longitudinal direction $x$, was calculated for every position within the volume of the board. The axial dynamic MOE was used to set/adjust board specific values of the MOEs and shear modulus.

(d) The edgewise bending stiffness for each position $x$ along the board was calculated by integration over the cross sectional area as illustrated in Fig. 2c, i.e. as

$E I_{z}(x)=\iint E_{x}(y-\bar{y})^{2} d A$

where,

$\bar{y}=\iint E_{x} y d A / \iint E_{x} d A$

(e) A high-resolution bending MOE profile

$E_{b}(x)$ was calculated as,

$E_{b}(x)=\frac{E I_{z}(x)}{t h^{3} / 12}$ 


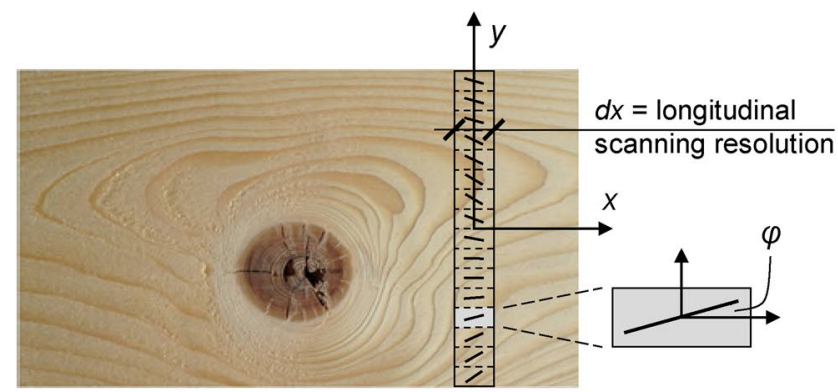

(a)

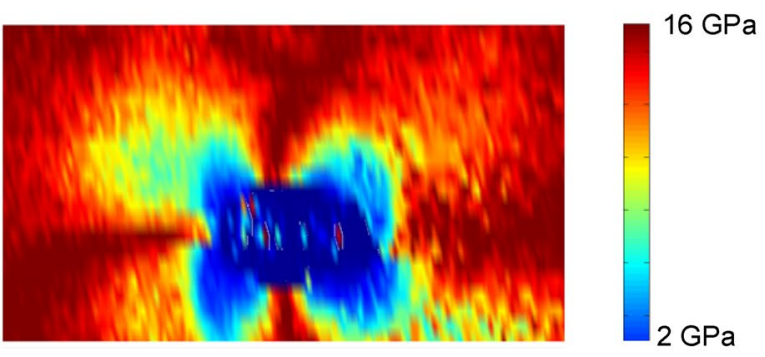

(b)

Fig. 2 a Local fibre orientation scanned on a board surface by means of a row of laser dots, $\mathbf{b}$ distribution of longitudinal MOE around the exhibited knot, $\mathbf{c}$ segment of length $d x$ for which the edgewise bend-

where $t$ and $h$ are the thickness and depth, respectively, of the board cross sections. Figure $2 \mathrm{~d}$ shows a calculated MOE profile, established by calculating a moving average of the high-resolution-profile over a length of $90 \mathrm{~mm}$. The lowest bending MOE along the profile was the suggested IP to bending strength.

This short summary of the procedure is sufficient to point out, for further discussion, two assumptions made. The first one concerns the quite simple representation of fibre orientation locally within the board. In reality, of course, the fibre orientation within the board is not identical to the one on the surface. Instead, this orientation depends on the occurrence and orientation of knots, which in turn depends on the position of the pith in relation to the board cross section. The other assumption is inherent in the calculation of the bending MOE according to Eqs. (1)-(3). In reality, there is more compliance/flexibility in the board than what the stiffness obtained by integration over vertical cross sections implies. In a study carried out by $\mathrm{Hu}$ et al. (2015), digital image correlation (DIC) technique was applied to one wide face surface of a board subjected to bending, with the purpose to establish, on an experimental basis, a bending MOE profile to use for comparison of a bending MOE profile calculated according to Olsson et al. (2013). The comparison showed that the calculated bending MOE profile underestimated the loss of bending stiffness caused by knots and fibre distortions in sections that
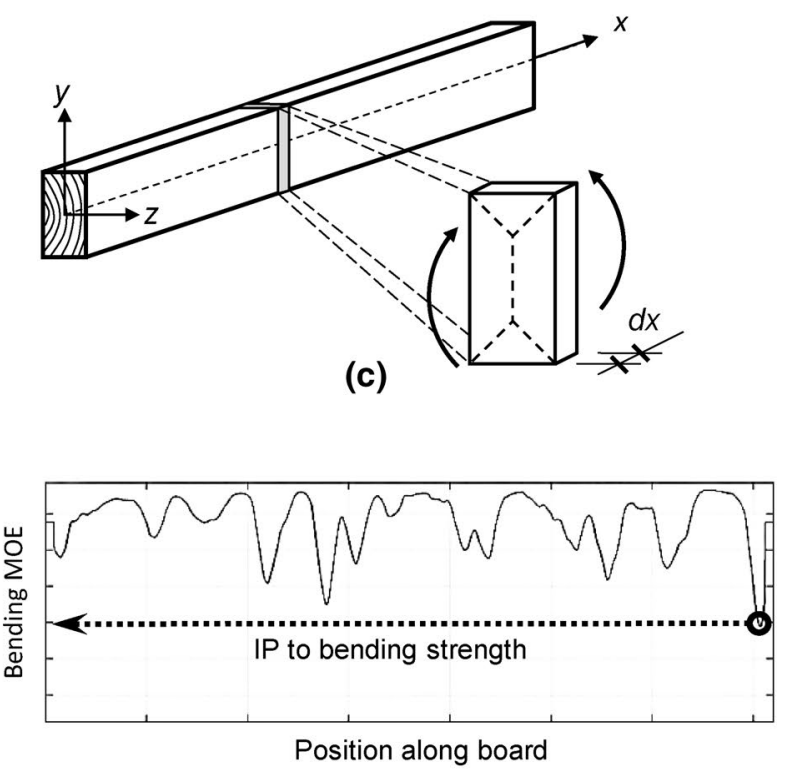

(d)

ing stiffness is calculated by integration over its cross section, $\mathbf{d}$ a bending MOE profile obtained by considering all the segments of the board

contained intricate knot clusters. Below, models that are based on integration over cross-sections for calculation of bending MOE (Eqs. 1-3) are termed 1D models.

\subsection{Aim and scope}

The purpose of the present study was to examine the possibilities of establishing accurate bending MOE profiles based on the knowledge of fibre orientation obtained by tracheid effect laser scanning in combination with knowledge of axial dynamic MOE, i.e. to investigate and elaborate on the concept of the machine strength grading method proposed by Olsson et al. (2013). This includes development and assessment of an improved fibre direction model and of a mechanical model that does not suffer from the simplifying assumption of earlier models, aiming at models that enable more accurate bending MOE profiles, and at the quantification of the significance of the simplifying assumptions pointed out above. More specifically, the following aims and actions are included in the paper:

1. Development of a model of fibre orientation of the interior of the board. This model will include information regarding the orientation of knots within a board. 
2. Development of a 3D FE model that allows for the elastic deformation/compliance of the 3D structure, i.e. a model that avoids the inherent constraints of simpler 1D models.

3. Obtaining strain fields and bending stiffness profiles using DIC techniques for boards in four-point bending tests.

4. Evaluation of the abilities and limitations of 1D and 3D models, respectively, and of two different models for representation of fibre orientation of the interior of the board, by comparing strain fields and bending MOE profiles that originate from experiments and simulations, respectively. The simulations are based on four different models.

\section{Material and introductory investigation of specimens}

The study comprised two boards of Norway spruce [Picea abies (L.) H. Karst], denoted board B1 and B2. The boards were of dimensions $45 \mathrm{~mm} \times 145 \mathrm{~mm} \times 4550 \mathrm{~mm}$ and the moisture content, at the time of laboratory testing, was $12 \%$. The board density, $\rho$, was calculated on the basis of the dimensions and weight of the boards at $12 \%$ moisture content. Furthermore, the first axial resonance frequency, $f_{\mathrm{a} 1}$, was determined by means of dynamic excitation using an impact hammer, vibration response captured by a microphone, and fast Fourier transformation using a spectrum analyser. The axial dynamic MOE of the boards were established as,

$E_{\mathrm{dyn}}=4 \rho \cdot f_{a 1}^{2} \cdot L^{2}$ where $L$ is the length of the board.

The location of the pith of each of the logs from which the two boards were sawn was determined in relation to the centre of the end cross-sections of the boards. This was done using a simple tool consisting of a transparent plastic sheet on which a number of concentric arcs and a 2D grid were drawn, see Fig. 3a. The position of the pith was determined by fitting the annual rings visible on the end surfaces of the boards to the concentric arcs on the plastic sheet, as illustrated in Fig. 3b. For each board, the position of the pith was determined as the mean value of the pith position of the two end surfaces. Table 1 shows results regarding board density, axial resonance frequency, dynamic MOE and determined position of pith for the two boards. Note that the pith, for both boards, was located outside the board cross-section.

The introductory investigation also consisted of laser scanning of the boards and utilization of the tracheid effect,

Table 1 Board dimension, density, axial resonance frequency, dynamic MOE and location of pith of investigated boards

\begin{tabular}{lll}
\hline Board properties & \multicolumn{2}{l}{ Value } \\
\cline { 2 - 3 } & $\mathrm{B} 1$ & $\mathrm{~B} 2$ \\
\hline Dimension $(\mathrm{mm})$ & $45 \times 145 \times 4550$ & $45 \times 145 \times 4550$ \\
Board density, $\rho\left(\mathrm{kg} / \mathrm{m}^{3}\right)$ & 458 & 464 \\
Axial frequency, $f_{\mathrm{a} 1}(\mathrm{~Hz})$ & 548 & 559 \\
Dynamic MOE, $E_{\mathrm{dyn}}(\mathrm{GPa})$ & 11.4 & 12.0 \\
Pith location $(y, z)(\mathrm{mm})^{\mathrm{a}}$ & & \\
Top end & $(-0.5,72.5)$ & $(-7.5,45.5)$ \\
Root end & $(-7.5,82.5)$ & $(-7.5,37.5)$ \\
Average & $(-3.5,77.5)$ & $(-7.5,41.5)$ \\
\hline
\end{tabular}

${ }^{a}$ Coordinates according to coordinate system defined in Fig. 3b

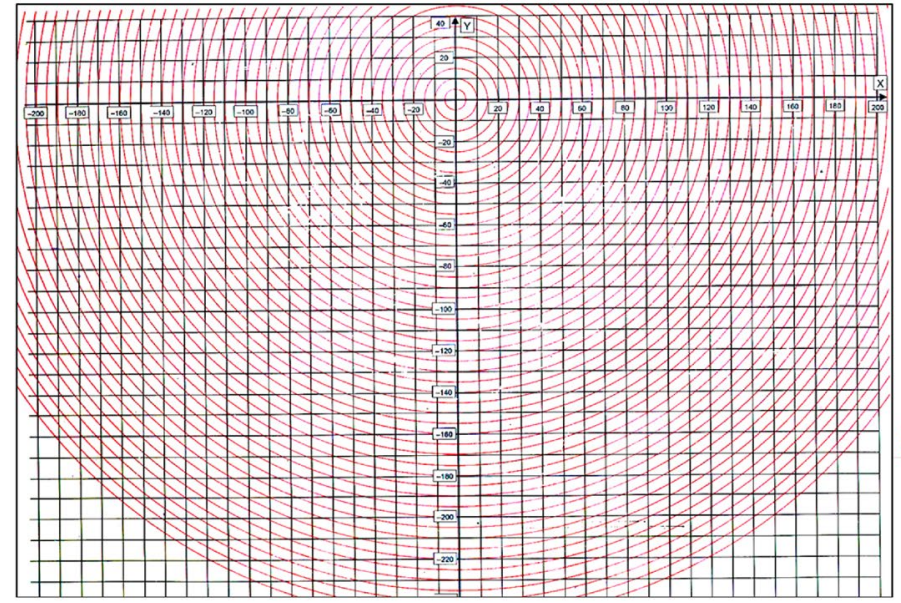

(a)

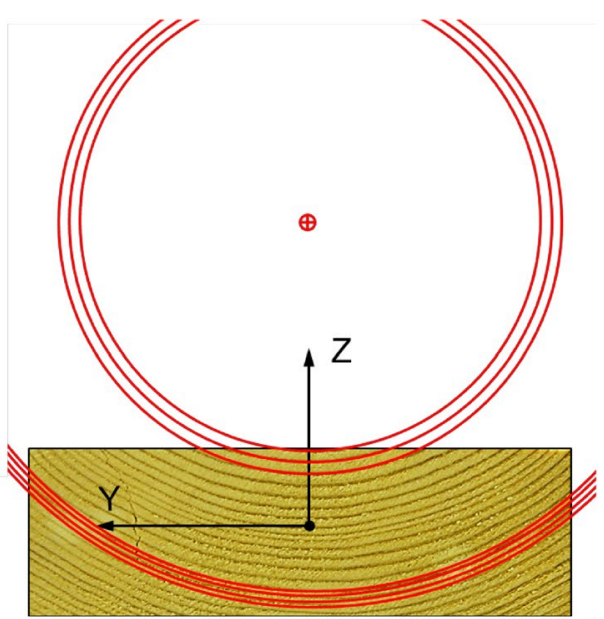

(b)

Fig. 3 a Transparent plastic sheet used to determine the location of the pith and $\mathbf{b}$ illustration of principle for determination of the location of the pith, relative to the centre of the board cross-section 
as described in the introduction, in order to obtain knowledge of in-plane fibre orientation on the surfaces. For this purpose, an optical scanner of make WoodEye 5 (WoodEye 2017) was used. Boards are fed through the scanner in longitudinal direction, and each of the four surfaces are illuminated by a row of laser rays. Each such row, which is achieved by a diffraction splitter through which a laser ray is divided into several rays, is oriented in the transversal board direction and exposing the whole width of the surface. Due to the tracheid effect, each ray illuminating the surface results in an elliptically shaped laser spot and pictures of these spots are sampled by cameras at a certain frequency. The resolution obtained in the longitudinal board direction is partly dependent on the sampling frequency, but also on the speed with which the board is fed through the scanner; the higher the speed, the lower the resolution. In the current investigation, a speed of $100 \mathrm{~m} / \mathrm{min}$ was used which gave a resolution of $1 \mathrm{~mm}$ in the longitudinal direction. The resolution in the transversal direction is determined by the design of the applied diffraction splitter, which in this investigation gave a transversal resolution of $4.4 \mathrm{~mm}$. Thus, a grid of in-plane fibre angles with a resolution of $1 \mathrm{~mm} \times 4.4 \mathrm{~mm}$ was achieved. The fibre orientation data obtained herein is comparable, regarding resolution, with the data obtained and utilized by for example Olsson et al. (2013) and Hu et al. (2015).

\section{Method}

The method used consisted of modelling and calculations, presented in Sects. 3.1-3.4, and laboratory investigations presented in Sect. 3.5.

\subsection{Modelling of fibre orientation and material directions within members}

The angle between the longitudinal direction of the board and the in-plane component of the fibres, locally on a board surface, was determined and set to represent the angle between the local fibres and the longitudinal direction of the board.

The angle between fibres and the longitudinal direction of the board were thus determined for a grid corresponding to the scanning resolution on each of the four sides of the board. The corresponding angles of the interior of the board were then to be determined and for this purpose, two alternative fibre angle models, hereinafter named FAM1 and FAM2, were established. FAM1, illustrated in Fig. 4a, is identical with the fibre angle model suggested by Olsson et al. (2013). In this model, the fibre angle determined in a position on a board surface (red dots in Fig. 4a) is used to represent the fibre angle in every position (shaded volume

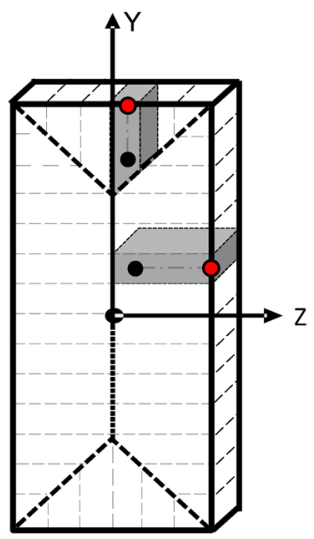

(a)

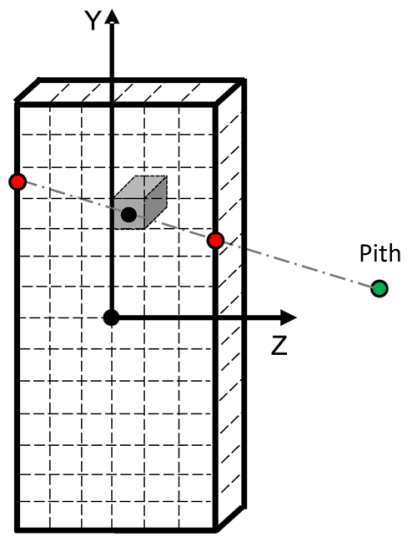

(b)
Fig. 4 Models for representation, in the interior of the board, of the angle between the fibre direction and the longitudinal board direction on the basis of known such angles on the board surfaces; a illustration of FAM1 and b illustration of FAM2

in Fig. 4a) from the surface to a certain depth into the board (black dots in Fig. 4a). FAM2, illustrated in Fig. 4b, requires knowledge of the location of the pith of the tree from which the board is cut. In this model the fibre angle in a position within the board (black dot in Fig. 4b) is determined by the fibre angles on board surfaces at positions (red dots in Fig. 4b) where the surfaces intersect with a straight line drawn from the pith (green dot in Fig. 4b) through the position (black dot in Fig. 4b) where the fibre angle shall be determined. The line intersects with two positions on board surfaces (red dots in Fig. 4b) and the fibre angle in the position within the board (black dot in Fig. 4b) is determined by linear interpolation of the fibre angles at the points of surface intersection.

The angle between longitudinal direction of the board and the local fibres is thus determined for a set of positions, i.e. a 3D grid of the board volume, according to FAM1 and FAM2, respectively.

Finally, the assumption was made for both models that the longitudinal-tangential plane ( $l t$-plane) of the wood material coincides with the $x y$-plane where the $x$-axis follows the longitudinal direction of the board and the $y$-axis follows the depth direction of the board, as shown in Fig. 4a, b. This means that the radial direction was assumed to be parallel with the $z$ direction of the board. Note that in-plane fibre angles observed on the narrow faces of the board, i.e. on surfaces that are actually parallel to the $x z$-plane are, nevertheless, regarded as angles in the $l t$-plane in the models. None of the FAM models is thus a complete 3D fibre orientation model (i.e. none of them provide a realistic distinction between radial and tangential material directions) but both provide representations of the angle between fibre direction and longitudinal board direction within the volume of the board. The advantage of FAM2, in 
comparison with FAM1, is that it takes the natural direction of knots, which is always from the pith and outward, into account. As described, this is done by means of interpolation between two positions on the surfaces (to assign a fibre angle in a position of the interior of the board) in the direction from the pith and outward, as illustrated in Fig. 3b. Thus, FAM2 should give a more realistic representation of the angle between fibres and the longitudinal board direction of the inner of the board volume than what FAM1 does.

\subsection{Modelling of material properties}

Regarding material properties, an orthotropic linear elastic material model with nine parameters was used and a set of nominal parameter values, representative for Norway spruce from Dinwoodie (2000), was adopted, see the second column in Table 2. Adjusted values of the six stiffness parameters were then determined for each individual board, by multiplying a board specific scaling factor, $\mu$, with each of $E_{\mathrm{l}}, E_{\mathrm{r}}, E_{\mathrm{t}}, G_{\mathrm{r}}$, $G_{\mathrm{tl}}$ and $G_{\mathrm{tr}}$ The scaling factor $\mu$ was determined in the same way as by Olsson et al. (2013), who presented a 1D model for establishment of MOE profiles as described in Sect. 1.4, including the FAM1 model presented in Sect. 3.1. The following description of the procedure for how to determine $\mu$ is thus an extension of the description of step (c) of Sect. 1.4.

Based on the nominal material parameters and FAM1, $\mathrm{E}_{\mathrm{x}}(x$, $y, z)$ was calculated for every position within the board. This included transformation of material parameters from the $l r t$ to the $x y z$-coordinate systems (see e.g. Ormarsson 1999). A simple 1D FE model, where the board was discretized into $n$ elements, was created. The longitudinal stiffness of each such element was calculated as,

$\frac{E A(x)}{L_{e}}=\frac{\iint \bar{E}_{x} \cdot d y d z}{L_{e}}$

where $L_{\mathrm{e}}$ is the element length, in this case about $1 \mathrm{~cm}$ in $x$ direction, $x$ represents an arbitrary position along the $x$ axis,

Table 2 Material parameters employed in this research; the nominal values originate from Dinwoodie (2000)

\begin{tabular}{lll}
\hline Parameter & Nominal values & Adjusted values \\
\hline$E_{l}$ & $10,700 \mathrm{MPa}$ & $10,700 \mu \mathrm{MPa}$ \\
$E_{r}$ & $710 \mathrm{MPa}$ & $710 \mu \mathrm{MPa}$ \\
$E_{t}$ & $430 \mathrm{MPa}$ & $430 \mu \mathrm{MPa}$ \\
$G_{r l}$ & $500 \mathrm{MPa}$ & $500 \mu \mathrm{MPa}$ \\
$G_{t l}$ & $620 \mathrm{MPa}$ & $620 \mu \mathrm{MPa}$ \\
$G_{t r}$ & $24 \mathrm{MPa}$ & $24 \mu \mathrm{MPa}$ \\
$v_{t l}$ & 0.025 & 0.025 \\
$v_{t r}$ & 0.31 & 0.31 \\
$v_{l r}$ & 0.38 & 0.38 \\
\hline
\end{tabular}

i.e. $x \in\left(0: L_{\mathrm{e}}: L\right)$, and $\bar{E}_{x}$ is the local average MOE in $x$ direction over the distance $L_{\mathrm{e}}$. Thus, the stiffness of the board in longitudinal direction was represented by these $n$ elements.

Furthermore, it was assumed that the mass of the considered board was evenly distributed along it. Then the first axial resonance frequency, $\hat{f}_{a 1}^{2}$, was calculated by performing eigenvalue analysis on the thus established 1D model and $\mu$ was calculated as:

$\mu=\frac{f_{a 1}^{2}}{\hat{f}_{a 1}^{2}}$

where $f_{\mathrm{a} 1}$ is the experimentally assessed resonance frequency of the same board. This means that if $E_{\mathrm{x}}(x, y, z)$ is calculated on the basis of the material parameters of the third column in Table 2, rather than on the material parameters of the second column in Table 2, the calculated resonance frequency will be identical to the experimentally assessed one.

\subsection{Calculation of bending MOE on the basis of integration over cross sections}

The 1D model for calculation of bending MOE along the board based on the integration over cross sections (IOCS) as described in Sect. 1.4 can be used in combination with FAM1, as by Olsson et al. (2013), or in combination with FAM2, see Sect. 3.1. Below, the two models are termed IOCS_FAM1 and IOCS_FAM2, respectively.

\subsection{Calculation of bending MOE on the basis of 3D FE models of boards}

A 3D FE model avoids the mechanical constraints that according to the last paragraph of Sect. 1.4 are inherent in the IOCS models. Therefore, a 3D FE model was developed and used in a simulation of a load case of pure bending. Based on calculated longitudinal strains, a bending MOE profile, comparable to those obtained based on the IOCS models, was calculated. A detailed description of the procedure follows.

\subsubsection{D FE model and simulation of pure bending}

3D models of boards were established and analysed using the commercial software MATLAB (from MathWorks Inc, Natick, Massachusetts USA) and ABAQUS (from Dassault Systèmes, Vélizy-Villacoublay, France). The FE model consisted of 8-node linear brick, full integration elementselement C3D8 of ABAQUS. The element size used was 5 $\mathrm{mm} \times 5 \mathrm{~mm} \times 5 \mathrm{~mm}$ and a convergence study showed that this element mesh was sufficient for the present purpose. 
The basis for material directions locally in the board volume was FAM1 and FAM2, respectively, as defined in Sect. 3.1. The material of the model was orthotropic as described in Sect. 3.2 and the employed values of material parameters were those defined in the third column in Table 2, i.e. with $\mu$ determined for each of the two boards, B1 and B2. This means that the IOCS_FAM1 model was also applied when determining the material parameters of the FE model.

The model of the board comprised the entire length of $4550 \mathrm{~mm}$. Constraints were applied such that the end sections had to remain plane as the board was deformed. A constant bending moment, $M_{\mathrm{z}}$, was applied by external loads/ bending moments acting at each end of the board. Furthermore, regarding practical implementation, material directions in positions corresponding to the element grid, and based on FAM1 and FAM2, respectively, were prepared in MATLAB. This data was then imported to ABAQUS via an analysis Input file. Thereafter the FE simulation was carried out using ABAQUS/standard and data of calculated 3D nodal coordinates and corresponding displacements were exported to MATLAB, which enabled post-processing of calculated displacements in MATLAB.

\subsubsection{Assessment of strain fields and bending MOE}

The 3D coordinates and displacements from the FE modelling and simulation were used to calculate the local engineering strains in the longitudinal direction of the board with a resolution corresponding to the element mesh. In order to reduce any noise of the strain field (remember that the material directions locally depend on the fibre orientation determined on the basis of a single laser dot, see Sects. 1.3-1.4) a lower resolution strain field was calculated on the basis of the high resolution strain field. The strain in a certain element was calculated as the average strain of a surrounding area of about $20 \mathrm{~mm} \times 20 \mathrm{~mm}$ in the $x y$-plane and denoted $\boldsymbol{\varepsilon}_{\mathrm{x}}(x, y, z)$. In the next step, a desired longitudinal resolution of calculated bending stiffness of the board, represented by a longitudinal distance $L_{\mathrm{r}}$ (e.g. $50 \mathrm{~mm}$ ), was determined. The average longitudinal strains over the surrounding distance $L_{\mathrm{r}}, \boldsymbol{\varepsilon}_{\mathrm{x}, \mathrm{r}}\left(x_{\mathrm{p}}, y, z\right)$ was calculated for positions $x_{\mathrm{p}}$, i.e. at every position of the $5 \mathrm{~mm}$ mesh/grid of the cross section. Note that the distance between $x_{\mathrm{p}}$ and $x_{\mathrm{p}+1}$ is also $5 \mathrm{~mm}$ due to the chosen element grid. In clear wood sections, $\boldsymbol{\varepsilon}_{\mathrm{x}, \mathrm{r}}\left(x_{\mathrm{p}}, y\right.$, $z$ ) is close to a linear function of $y$, and almost independent of $z$, but this is not the case in sections containing knots. $\boldsymbol{\varepsilon}_{\mathrm{x}, \mathrm{r}}\left(x_{\mathrm{p}}, y, z_{\mathrm{c}}\right)$, where $z_{\mathrm{c}}$ is a selected constant position on the $z$ axis, was however approximated with a linear function of $y, \bar{\varepsilon}_{x, \mathrm{r}}\left(x_{p}, y, z_{c}\right)$, determined by linear regression performed on $\boldsymbol{\varepsilon}_{\mathrm{x}, \mathrm{r}}\left(x_{\mathrm{p}}, y, z_{\mathrm{c}}\right)$.

The cross sectional bending stiffness of the board was finally determined based on $\bar{\varepsilon}_{x, \mathrm{r}}\left(x_{p}, y, z_{c}\right)$, i.e. for a given plane of $\mathrm{z}=\mathrm{z}_{\mathrm{c}}$, at every position $x_{\mathrm{p}}$ along the board, the cross sectional bending stiffness was calculated as:

$E I_{z, \mathrm{r}, \mathrm{c}}\left(x_{p}\right)=\frac{M_{z}}{\bar{\varepsilon}_{x, \mathrm{r}}\left(x_{\mathrm{p}}, y_{1}, z_{\mathrm{c}}\right)}\left(y_{0}-y_{1}\right)$

where $M_{\mathrm{z}}$ is the applied constant bending moment, $y_{0}$ is the position on the $y$-axis where $\bar{\varepsilon}_{x, \mathrm{r}}\left(x_{p}, y_{0}, z_{c}\right)=0$ and $y_{1}$ is any other position along the $y$-axis. Thus, $E I_{\mathrm{z}, \mathrm{r}, \mathrm{c}}\left(x_{\mathrm{p}}\right)$ is the calculated edgewise bending stiffness, of longitudinal resolution $L_{\mathrm{r}}$, calculated based on strains in the $x y$-plane where $z=z_{\mathrm{c}}$. Finally, the corresponding bending MOE, $E_{\mathrm{b}}(x)$, was calculated by Eq. (3), with $E I_{\mathrm{z}}(x)=E I_{\mathrm{z}, \mathrm{r}, \mathrm{c}}\left(x_{\mathrm{p}}\right)$. Below, the 3D FE model is denoted FE_FAM1 when used in combination with FAM1, and FE_FAM2 when used in combination with FAM2.

Figure 5a shows a calculated, using FAM1, strain field $\boldsymbol{\varepsilon}_{\mathrm{x}}\left(x, y, z_{\mathrm{c}}\right)$ for $z_{\mathrm{c}}=22.5 \mathrm{~mm}$, i.e. the strains in longitudinal direction on one wide surface of a $600 \mathrm{~mm}$ long part of board B1. Thus, the strains displayed represent average strains over surrounding areas about $20 \mathrm{~mm} \times 20 \mathrm{~mm}$. The inclined straight lines plotted on top of the strain plot represent $\bar{\varepsilon}_{x, \mathrm{r}}\left(x_{p}, y, z=22.5\right)$, i.e. lines of linear regression calculated on the basis of longitudinal strains of vertical sections. The distance between two adjacent lines was determined by the element mesh, i.e. $5 \mathrm{~mm}$. The strains on which these lines are based were average strains over a distance of $50 \mathrm{~mm}$, i.e. $L_{\mathrm{r}}$ was $50 \mathrm{~mm}$. In Fig. $5 \mathrm{~b}$, c, black and red dots represent calculated average strains, and solid lines are lines of regression calculated based on the strains for two vertical sections of the board, one free of knots (black line in Fig. 5a, b) and the other with knots (red line Fig. 5a, c). In the knot free section, the black dots follow the straight line very well, with a coefficient of determination $R^{2}=0.999$, whereas in the knotty section the strain values clearly deviate from the line of regression, the coefficient of determination $R^{2}=0.915$. Figure $5 \mathrm{~b}, \mathrm{c}$ also show that the slopes of the regression lines, representing the bending stiffness, are larger in the section free of knots, i.e. bending stiffness in a clear wood section is higher than in a section with knots. Figure $5 \mathrm{~d}$ shows the bending MOE variation in the exhibited part of the specimen, determined on the basis of all the lines of linear regression shown in Fig. 5a.

\subsection{Laboratory measurements as the basis for strain fields and bending MOE}

The boards B1 and B2 were subjected to four-point bending in laboratory, and non-contact DIC was performed to detect displacement of the board surfaces and to obtain strain fields comparable to those obtained based on the FE models. Then local bending MOE was calculated based on 


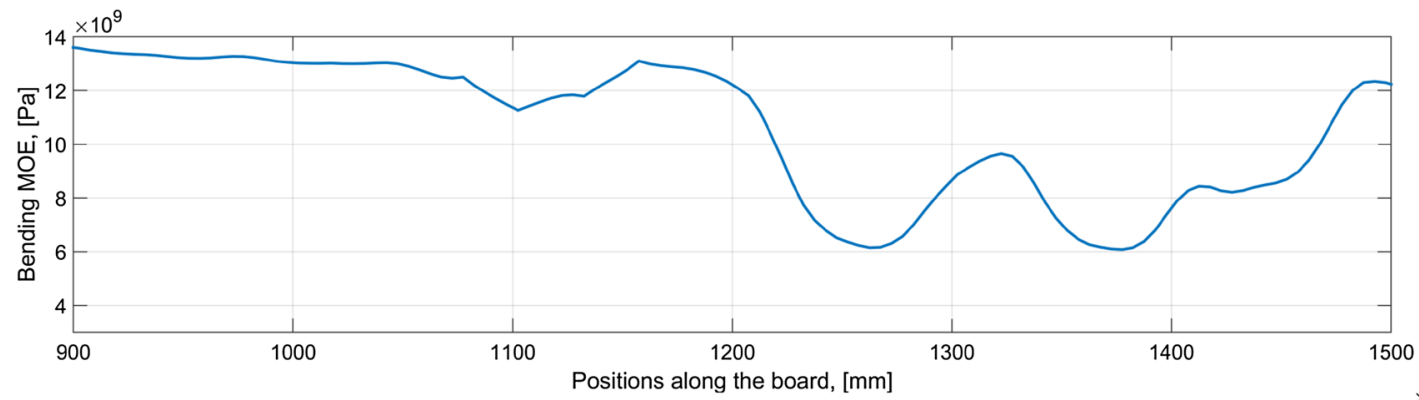

(d)

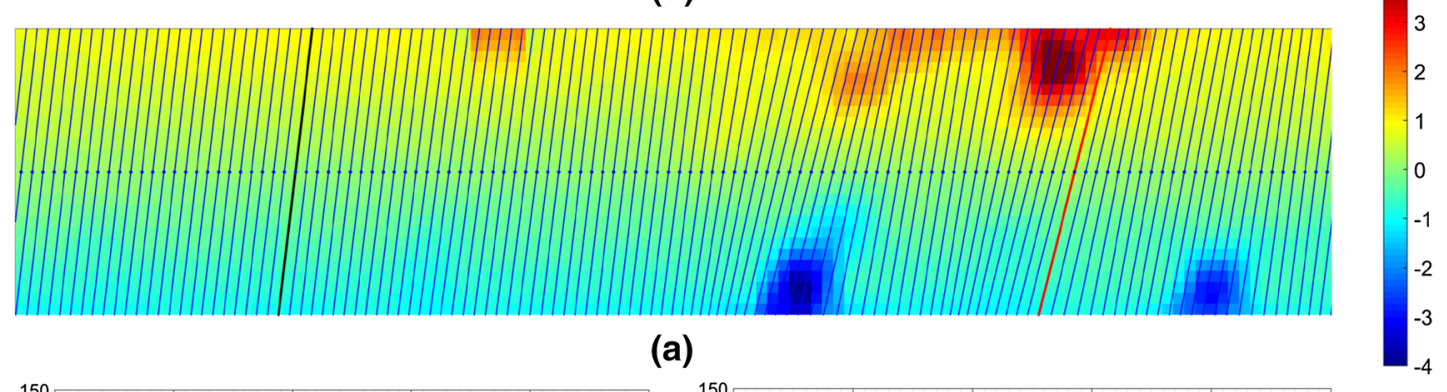

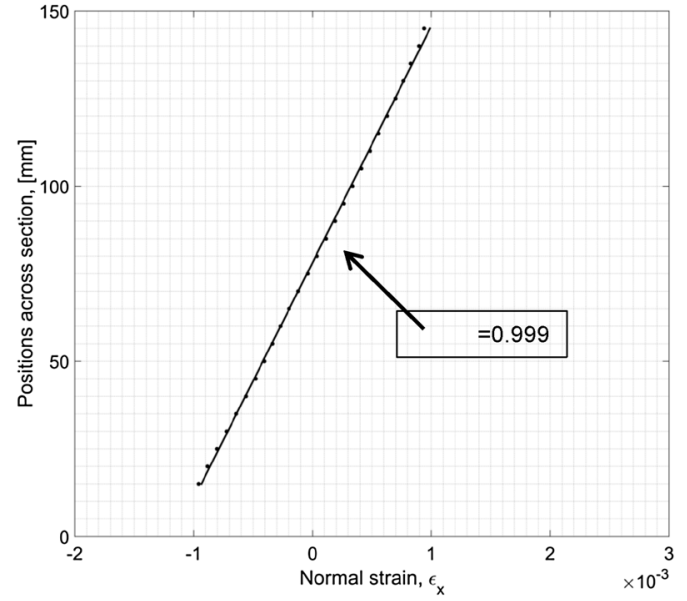

(b)

Fig. 5 a Examples of strain distribution calculated using the FE model in combination of FAM1, where each strain value displayed represents a mean value of the surrounding area about $20 \mathrm{~mm} \times 20$ $\mathrm{mm}$. Lines of regression of longitudinal strains over vertical cross sections drawn on top of the strain plot. Two lines are highlighted in

these experimentally obtained strain fields in a similar way as described in Sect. 3.4.2 for strains calculated on the basis of results of the FE simulation. A description of the equipment used and procedure performed follows.

\subsubsection{Equipment}

Four-point bending of the two boards was achieved using a testing machine that is a manually controlled hydraulically actuated system including two load cells, each having capacity of $\pm 100 \mathrm{kN}$. Using the testing machine, it is possible to test beam specimens with lengths up to $6 \mathrm{~m}$,

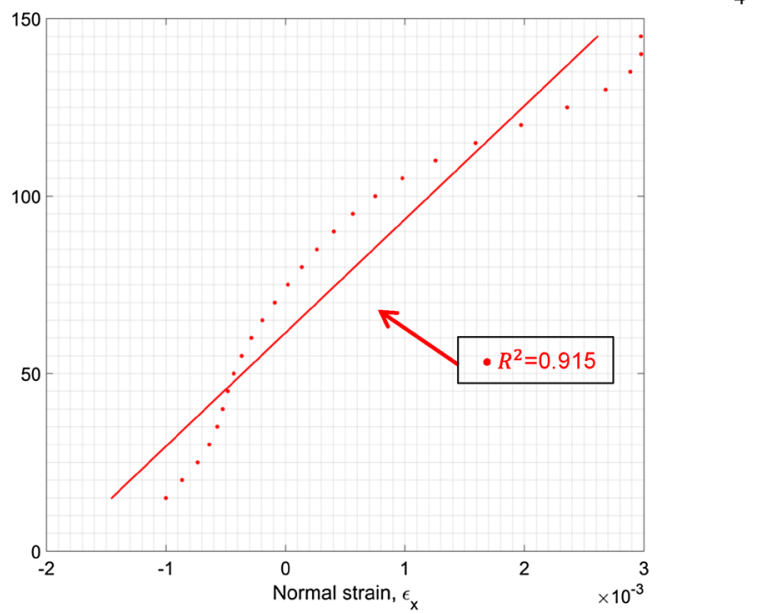

(c)

black and red. These are enlarged in $\mathbf{b}$ and $\mathbf{c}$ respectively, and shown along with the original strain values on which they are based. The corresponding $R^{2}$-values indicate to what extent the strains along a vertical line at a certain coordinate $z=z_{\mathrm{c}}$ comply with the straight lines. d Bending MOE profile based on the regression lines of a

and the positions of loading and support are fully adjustable along the length of specimens. At the loading and the support positions, small steel plates, $100 \mathrm{~mm} \times 150 \mathrm{~mm}$ in size, are attached to the specimen to prevent local indentations. In addition, lateral constraints are applied at the two loading positions to prevent lateral torsional buckling, and roller bearing setups are applied at the supports to allow an in-plane rotation.

By means of an optical measurement system called TRITOP (from Gesellschaft für Optische Messtechnik, Braunschweig, Germany) it is possible to establish 3D-coordinates for discrete points at pre-determined positions on a 
test object. Essential components for TRITOP measurement are (a) a photogrammetry camera, (b) coded reference point markers (RPMs), (c) scale bars, (d) a set of orientation crosses, and (e) uncoded RPMs. The components (b)-(d) are used to establish a global 3D coordinate system, which fits 1:1 to the test object and has a pre-aligned origin determined by one particular orientation cross within the set of crosses. Components (e), i.e. the uncoded RPMs, are usually the measuring targets. Performing a TRITOP measurement, the test object is photographed, by which pre-allocated RPMs are captured in many different views. To ensure high measurement accuracy, each image taken should include as many of the coded RPMs as possible spread over the test object. Using these images together with TRITOP software, it is possible to compute the 3D coordinates of the RPMs in the established global coordinate system. Typical output of a TRITOP measurement is a 3D coordinate system including desirable RPMs.

The DIC system ARAMIS (from Gesellschaft für Optische Messtechnik, Braunschweig, Germany), is appropriate for establishment of 3D-coordinates for full field of the test object. A 3D ARAMIS system is equipped with dual cameras with fixed relative positions. Digital images are taken before and during loading. By means of ARAMIS, and by applying a stochastic spray pattern to the test object, facets consisting of a certain number of pixels can be recognized in each pair of conjugated images from the two cameras. Furthermore, 3D coordinates can be allocated to the facets. An ARAMIS measurement is usually implemented by taking images at multiple load stages between which a certain load increment is applied. Thus, the movement of faces that due to the load increment occur on the objective's surface is captured and registered. Full-field ARAMIS measurement enables 3D displacements data with high spatial resolution. However, the resolution is dependent on the measuring volume; the smaller the measuring volume, the higher the spatial resolution.

DIC systems are frequently used for understanding the mechanical behaviour of timber including knots (e.g. Sjödin et al. 2006; Oscarsson et al. 2012, 2014), and for verification of timber models established computationally by given algorithms (e.g. Hu et al. 2015) or using FE method (Lukacevic and Füssl 2014).

\subsubsection{Procedure to obtain high resolution displacement fields}

To determine the strain fields and bending stiffness profiles based on laboratory experiments, the boards were subjected to a four-point bending test combined with DIC measurements. For the four-point bending, the boards were placed with the outer supports at the two ends of the boards and the point loads were applied at the centre span $3300 \mathrm{~mm}$ apart, see Fig. 6a. Thus, the test objects were the two wide surfaces of the boards, each having an area of about 145 $\mathrm{mm} \times 3300 \mathrm{~mm}$, see Fig. 6a. To capture the whole surface and ensure high-resolution measurement, each object surface was divided into six subareas for which six individual ARAMIS projects were performed. The TRITOP system was used to establish a global coordinate system and to relate all the six separate ARAMIS projects to this common global coordinate system. Thus, DIC data with a resolution of about 2.6 $\mathrm{mm} \times 2.2 \mathrm{~mm}$ in the transversal and longitudinal directions of the board was obtained for a board length of $3300 \mathrm{~mm}$. Figure 6 a shows the implementation of the jointed TRITOP and ARAMIS measurements. Measurement of each surface required one TRITOP project combined with six ARAMIS subprojects to be performed. The measuring procedure was initiated by the TRITOP project for establishment of the global coordinate system valid for the whole surface of the board. Figure $6 \mathrm{~b}$ illustrates the arrangement and preparation of the TRITOP project. A plywood was placed right behind the specimen. On the plywood a number of uncoded RPMs (small black dots on the plywood sheet in Fig. 6b), which were the target positions, were placed both above and below the specimen. In addition, coded RPMs, two scale bars and four orientation crosses were used. To ensure high quality data from the TRITOP project, plenty of coded RPMs were used and well spread on the specimen, as shown in Fig. 6b. Thus, 3D coordinates of the reference points on the plywood sheet were obtained by the TRITOP measurement. When the TRITOP measurement was done, the coded RPMs, the scale bars and the orientation crosses were removed and from now on, it was crucial to ensure the plywood sheet including the reference points to be kept in position until the subsequent ARAMIS measurements were accomplished.

Each ARAMIS subproject, covering an area of about $560 \times 500 \mathrm{~mm}^{2}$ as indicated by the shaded areas in Fig. $6 \mathrm{a}$, were ensured to capture at least three of the reference points on the plywood sheet. The reference points were the basis to make the subsequent coordinate transformation from the individual ARAMIS subproject to the global system of TRITOP. The point loads were increased in five steps, i.e. the five load stages shown in Table 3. To ensure full contact between the supports and the specimen, an initial point load of $500 \mathrm{~N}$ was applied. The nominal values of the point load in Table 3 are the load increments with respect to the initial load. At each load stage, the load level was kept constant and the cameras were placed at six positions along the board to complete the six subprojects. Thereafter, the load was increased to the next level according to the load stages. The procedure was repeated for all the load stages. The described measurement for each load stage took about $10 \mathrm{~min}$ to accomplish and deflection at the middle of the beam span was monitored using an extensometer that was placed at the bottom edge. The time history of the 


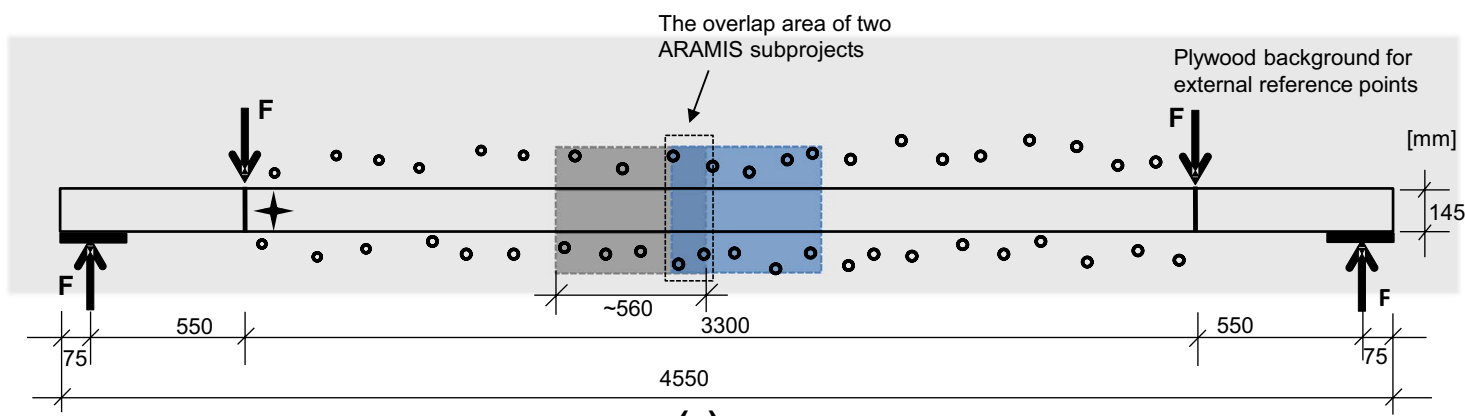

(a)

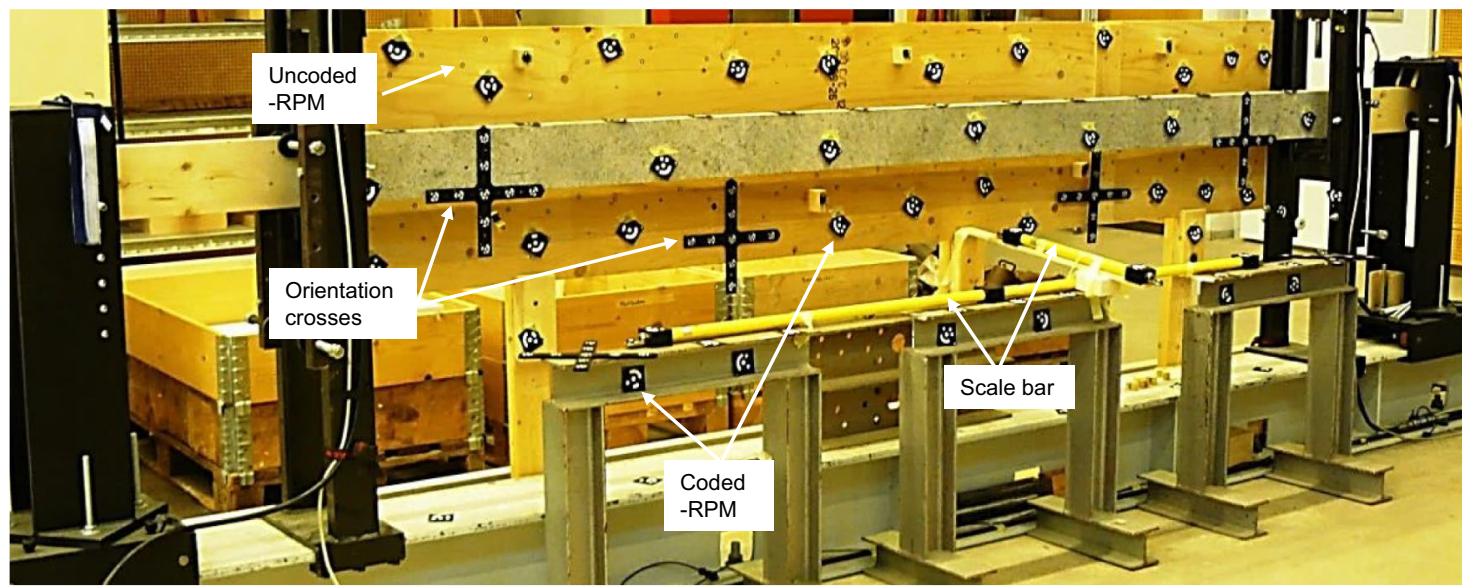

(b)

Fig. 6 a Schematic measurement setup of the bending test combined with DIC measurements. The middle $3300 \mathrm{~mm}$ of the specimen is exposed to a constant and pure bending moment and measured by six ARAMIS subprojects, each capturing a length of $560 \mathrm{~mm}$ along the

Table 3 Load stages of the ARAMIS measurements

\begin{tabular}{llllrr}
\hline Load stage no. & 0 & 1 & 2 & \multicolumn{1}{l}{3} & \multicolumn{1}{c}{4} \\
\hline Nominal point load $F(\mathrm{kN})$ & 0 & 1.0 & 2.0 & 3.0 & 4.0 \\
Nominal stress $\sigma(\mathrm{MPa})$ & 0 & 3.5 & 7.0 & 10.5 & 13.9 \\
\hline
\end{tabular}

mid-deflection showed that no creep occurred. When the measurement on one surface was completed, the board was unloaded and kept in place, while the plywood sheet was moved to the opposite side of the specimen to enable the measurement on the second wide surface by implementing a new TRITOP project coupled with six new ARAMIS subprojects in the same way. The described measurement procedures reveal that two uncoupled TRITOP systems were used for the two surfaces of the same board. However, the two systems were pre-aligned so that they shared the same origin regarding the $x$ - and $y$-coordinates. This was done by putting the particular orientation cross, i.e. the orientation cross determining the origin of the 3D-coordinate system, at the same physical position in the $x$ and $y$ directions on the two examined surfaces. board while the load was kept constant. b Arrangement of the TRITOP measurement, where coded RPMs, uncoded RPMs, orientation crosses and scale bars were used

The output data from the bending tests with DIC measurements are the $3 \mathrm{D}$ coordinates of facets in a 2D grid of $2.6(y) \times 2.2(x) \mathrm{mm}$ of the two wide surfaces of the boards. Regarding accuracy of the measured 3D coordinates, it was about $\pm 12 \mu \mathrm{m}$ in $x$ and $y$ directions, and about $24 \mu \mathrm{m}$ in $z$ direction. A smaller covering area of each subproject than the one used herein would allow higher accuracy. The data of load stage 0 and 4 , which were identical with the load levels applied to the FE simulation, were exported to MATLAB for assessment of strain fields and stiffness profiles.

\subsubsection{Strain fields and bending MOE based on measured displacements}

Strain fields were determined on the basis of displacements from the DIC system in a manner very similar to that on the basis of displacements from the FE simulations. Regarding strains from DIC, high resolution corresponds to low accuracy. Therefore, in order to obtain comparable strains with sufficiently high accuracy, strains with a resolution of 20 $\mathrm{mm} \times 20 \mathrm{~mm}$, i.e. average of the full-resolution strains over an area of $20 \mathrm{~mm} \times 20 \mathrm{~mm}$, were determined representing 
DIC results. For this resolution the accuracy of DIC strains became approximately $\pm 0.12 \%$. Note, however, that DIC and the experiment performed only give basis for calculation of strain fields at the wide surfaces of the board, i.e. for calculation of $\boldsymbol{\varepsilon}_{\mathrm{x}}\left(x, y, z_{\mathrm{c}}\right)$ where $z_{\mathrm{c}}= \pm 22.5 \mathrm{~mm}$. Similar to the procedure described in Sect. 3.4.2, linear, regression was performed on $\boldsymbol{\varepsilon}_{\mathrm{x}, \mathrm{r}}\left(x_{\mathrm{p}}, y, z_{\mathrm{c}}\right)$ resulting in $\bar{\varepsilon}_{x, \mathrm{r}}\left(x_{p}, y, z_{c}\right)$, only with the difference that the distance between two adjacent, evaluated positions in longitudinal direction, i.e. the distance between $x_{\mathrm{p}}$ and $x_{\mathrm{p}+1}$, being $2.2 \mathrm{~mm}$ (corresponding to the original, high resolution data of the DIC) rather than $5 \mathrm{~mm}$ (corresponding to the FE mesh). Then the bending stiffness $E I_{\mathrm{z}, \mathrm{r}, \mathrm{c}}\left(x_{\mathrm{p}}\right)$, i.e. a moving average bending stiffness over $50 \mathrm{~mm}$ (since $L_{\mathrm{r}}=50 \mathrm{~mm}$ ) was calculated according to Eq. (7), where $M_{\mathrm{z}}$ was now the constant bending moment applied to the laboratory experiment corresponding to the evaluated strain levels. Finally, $E_{\mathrm{b}}(x)$ was calculated according to Eq. (3).

\section{Results and discussion}

In this section, results regarding strain fields and bending MOE profiles of the boards are presented and discussed. Results presented are based both on the modelling and on the DIC measurements, and thus the suggested models are verified through comparison of the obtained results. The two wide surfaces of each specimen are herein denoted as Sa and $\mathrm{Sb}$, where Sa represents the surface close to the pith of the log. Regarding the comparison between the results of the modelling and the DIC measurements, similar results are achieved for the two specimens. To keep the paper concise, results presented below are based on specimen B2. Results of specimen B1 are shown in Online Resource 1 on the publisher's webpage.

\subsection{Strain distribution}

Results of longitudinal strains $\boldsymbol{\varepsilon}_{\mathrm{x}}$ occurring on the wide surfaces are presented using colour plots and section diagrams. Strain distribution over a surface is visualized by a colour plot, in which the colour indicates both the magnitude and the direction (being tensile or compressive) of the strain. A section is here defined by a horizontal or a vertical straight line displayed in the colour plots. Strains along such sections are plotted in diagrams against the corresponding positions.

Figure 7 shows the strain results at load stage 4 where (a) shows boards surface image of Sa valid for the $3300 \mathrm{~mm}$ long part of the specimen that was exposed to constant bending moment in the laboratory test. (b) shows the strain distribution valid for the corresponding surface calculated on the basis of displacements of DIC. Figure $7 \mathrm{c}-\mathrm{d}$ show the corresponding strain distribution calculated based on displacements from the FE simulation using FAM1 and FAM2, respectively. Figure 7e-h show the surface image of $\mathrm{Sb}$ and the corresponding strain field valid for $\mathrm{Sb}$ calculated based on displacements from DIC, FE_FAM1 and FE_FAM2, respectively. The strains displayed in Fig. 7 are average values over the surrounding area of $20 \mathrm{~mm} \times 20 \mathrm{~mm}$ and the strain levels are indicated by the colour bar to the right.

From the board surface image shown in Fig. 7, it can be observed that specimen B2 included numerous knots spread all over the board. If the smaller knots such as pin knots are disregarded, the remaining larger knots can be grouped into a number of knot clusters scattered along the board, and the sections between are more or less free from knots. As indicated in Fig. 7a, e, the upper edge of the specimen was exposed to tension and the lower edge was exposed to compression. When comparing the surface images with the corresponding strain plots, strain concentrations in areas with knots close to the edges emerge very clearly in all the strain plots. Another observation that can be made is that knots located in the middle of the board in the transversal direction do not result in strain concentrations because there is hardly any deformation occurring there in a pure bending condition.

Figure 8 shows an enlarged surface image and strain fields of the part of surface Sb highlighted in Fig. 7, in which (a) shows the enlarged surface image, (b)-(d) the enlarged strain plot of the highlighted part in Fig. 7 b-d, calculated on the basis of displacements from DIC, and from simulation using FE_FAM1 and FE_FAM2, respectively. In Fig. 8b-d, three horizontal and three vertical lines are drawn on the top of the colour plots. These lines represent sections 1, 2, 3 and $i$, ii, iii, respectively. The strain distributions along those sections are plotted in Fig. 8e, f for the horizontal and vertical sections, respectively.

From the board surface image in Fig. 8a, it is clear that this part of the board surface includes two large edge knots at both the tension and compression edge. Comparing the strain results calculated on the basis of the three sets of data, there is an obvious resemblance regarding both the pattern and the levels in Fig. 8b-d. Overall, the strain plots clearly show the strain concentrations in the areas where knots are visible on the surface image. However, Fig. 8b showing strains calculated using DIC indicate a smaller area of intensive strain concentration (area in dark red) close to the tension edge than what the strain results based on the FE simulations in Fig. 8c, d do. A close examination of Fig. 8a reveals that there was an initial crack in the upper knot. In the bending test, the crack opening was thus interpreted as an area of certain length with large strains, while the material that borders the crack indicates insignificant strain. However, the strain obtained based on the FE data indicates large positive strains over an area slightly larger than the knot itself, which is due to the fibre distortions and 


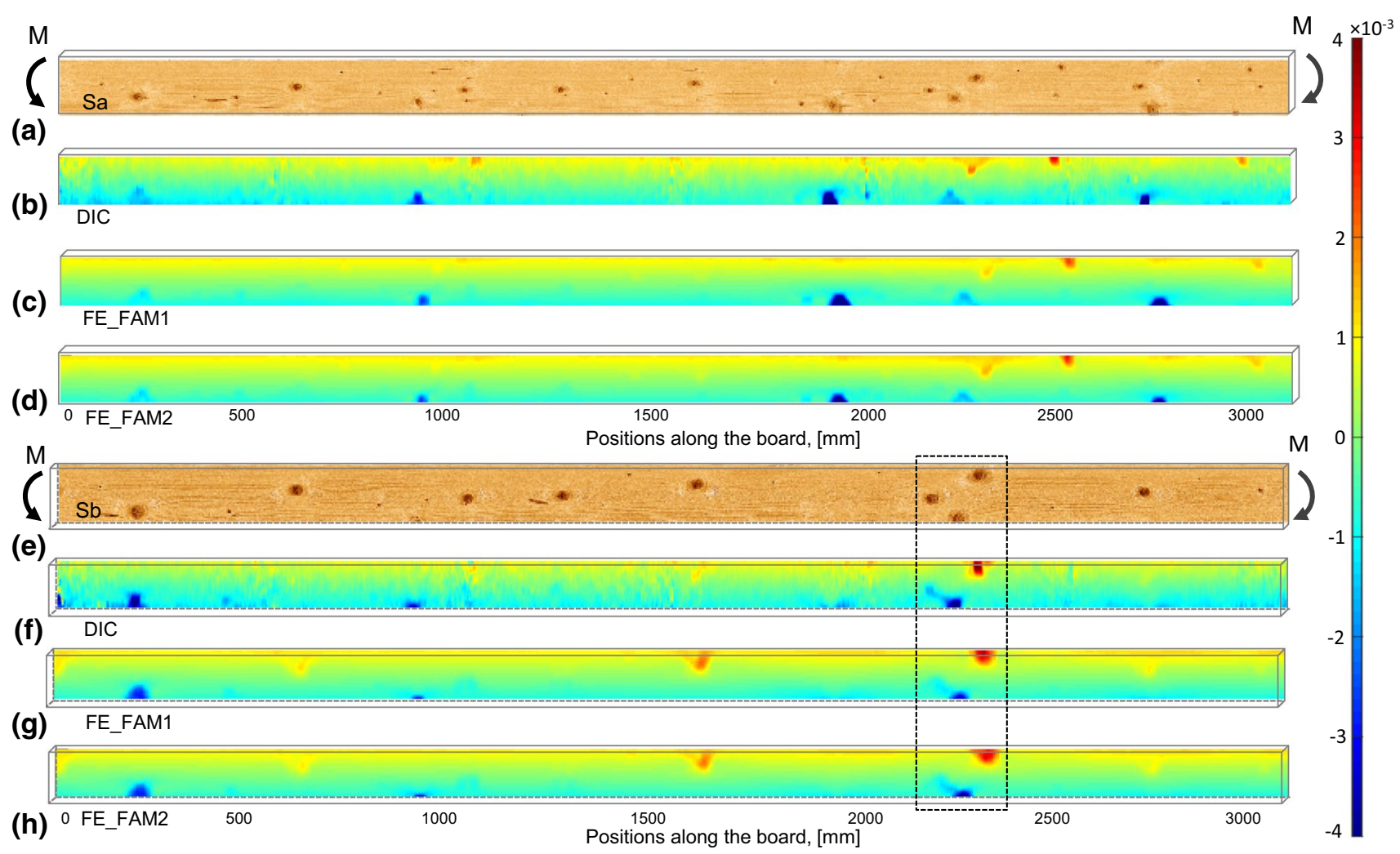

Fig. 7 Longitudinal strain $\boldsymbol{\varepsilon}_{\mathrm{x}}$ distribution obtained at load stage 4 for specimen B2. a Board surface image of Sa valid for the part which was exposed to the constant bending moment. b Strain field valid for the part of the specimen in a and calculated using the DIC data. c, d Strain field valid for the part of the specimen in a and calculated

the corresponding low local $\mathrm{MOE}\left(E_{\mathrm{x}}\right)$ also outside the knots themselves. In this respect, there is better agreement of strain concentration in the compressive zone in the area of the knot itself. However, in the compressive zone, in areas just above the edge knot to the left, slight differences in strain pattern/levels can be observed. Figure 8e-f quantitatively show the strain distribution in sections more or less free of knots, i.e. the sections 2, i, and in sections with knots, i.e. the sections 1,3 , ii and iii. When comparing the strains obtained from the FE data using FAM1 and FAM2, it seems like results of FE_FAM2 indicate a better agreement with the DIC results than what results from FE_FAM1 does. This holds, both when comparing colour plots and when comparing section diagrams and also holds for board B1, as shown in Online Resource 1.

\subsection{Bending stiffness profiles along members}

Bending MOE profiles, $E_{\mathrm{b}}(x)$, along the board $\mathrm{B} 2$ are presented in Fig. 9 where each value of each of the curves represents a mean bending MOE over a surrounding distance of $50 \mathrm{~mm}$ in the longitudinal direction of the board. Figure 9a using the data of FE_FAM1 and FE_FAM2, respectively. e-h Corresponding surface image and strain field results valid for surface $\mathrm{Sb}$. $\mathrm{An}$ area on the $\mathrm{Sb}$ surface with the corresponding strain distribution of the area is highlighted by a frame drawn in $\mathbf{e , f}$. The detailed strain information for this area is shown in Fig. 8

shows the surface image of Sa and the corresponding bending MOE profiles along the board, i.e. calculation of $E_{\mathrm{b}}(x)$ was based on Eq. (3) and Eq. (7) where $z_{\mathrm{c}}=22.5 \mathrm{~mm}$. Shown MOE profiles are thus based on strains, $\bar{\varepsilon}_{x, \mathrm{r}}\left(x_{p}, y, z_{c}\right)$, see Sect. 3.4.2, that originate from DIC (black line), FE_FAM1 (red line) and FE_FAM2 (purple line), respectively. Figure $9 \mathrm{~b}$ shows the surface image of $\mathrm{Sb}$ and the corresponding three MOE profiles, i.e. profiles calculated on the basis of strains $\bar{\varepsilon}_{x, \mathrm{r}}\left(x_{p}, y, z_{c}\right)$ where $z_{\mathrm{c}}=-22.5 \mathrm{~mm}$. Figure 9c shows MOE profiles that are average values of MOE profiles valid for different positions in the thickness direction of the board, i.e. for different values of $z_{c}$. The black curve, which is based on DIC data, shows the average of $E_{b}(x)$ calculated for $z_{c}$ $=22.5$ and $z_{\mathrm{c}}=-22.5$, while the red and purple curves, representing the FE models show the average of $E_{\mathrm{b}}(x)$ calculated on the basis of strains at $z_{\mathrm{c}}=-22.5, z_{\mathrm{c}}=-2.5, z_{\mathrm{c}}$ $=2.5$ and $z_{\mathrm{c}}=22.5$. This means that the curves of Fig. $9 \mathrm{c}$, representing the FE results, include knowledge of calculated strains in the interior of the board in a way that cannot be done for the profiles that are based on strains from DIC. In addition to the curves that represent DIC, FE_FAM1 and FE_FAM2, Fig. 9c also shows bending MOE profiles that 


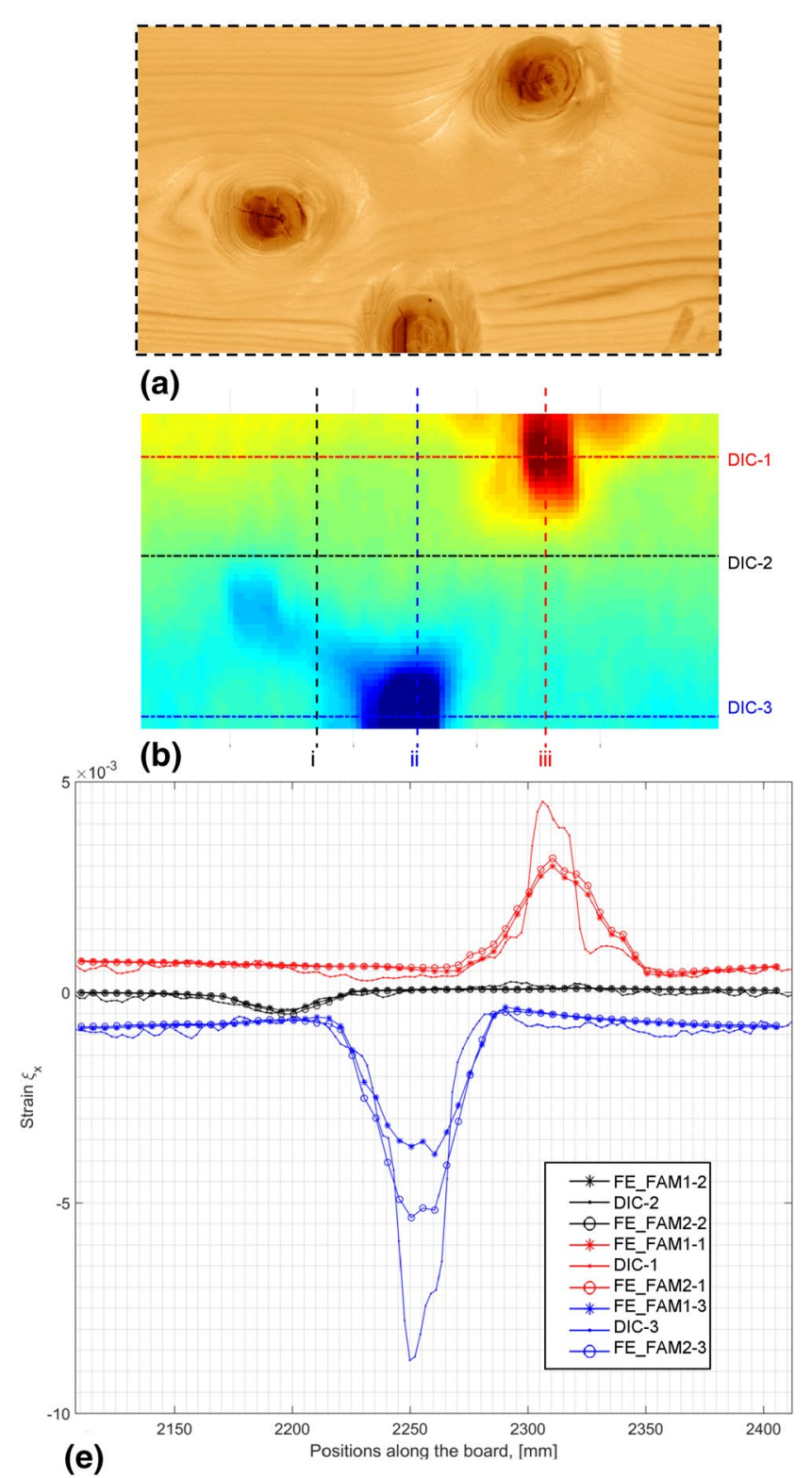

Fig. 8 Detailed strain information valid for the highlighted area in Fig. 7. a Enlarged surface image. b-d Enlarged strain distribution plot obtained based on displacements from b DIC, $\mathbf{c}$ simulation using FE_FAM1 and d simulation using FE_FAM2. Three horizon-

represent IOCS_FAM1 (blue) and IOCS_FAM2 (cyan), see Sect. 3.3. One part of the curves in Fig. $9 \mathrm{c}$ is highlighted and the enlarged view of this part is shown in Fig. 9d.

The profiles displayed in Fig. 9a, b show that the bending MOE drops to low levels in some positions along the board. Between these drops, the bending MOE is high and more or less constant. When comparing the profiles with the surface images, the drops appear at positions where knots or knot clusters are visible on the surface images. Moreover, the depths of the drops depend on the size, number and placement of the knot such that more and bigger knots close
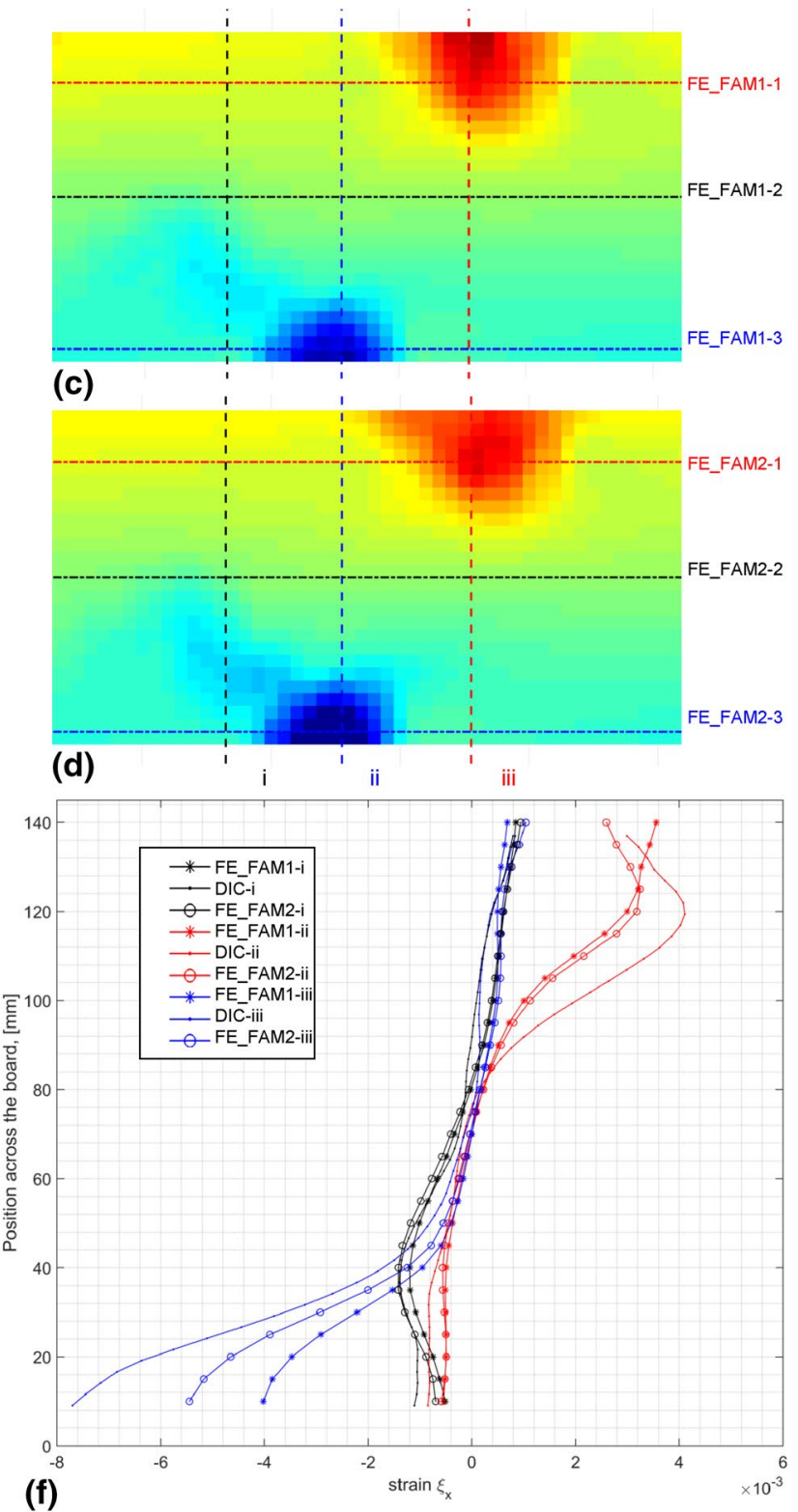

tal straight lines and three vertical straight lines, drawn in $\mathbf{b}-\mathbf{d}$, are marked 1, 2, 3 and i, ii, iii, respectively. e Section diagrams of strains, $\boldsymbol{\varepsilon}_{\mathrm{x}}$, along the horizontal Sect. 1, 2 and 3. f Section diagrams of strains, $\boldsymbol{\varepsilon}_{\mathrm{x}}$, along the vertical sections i, ii and iii

to the edges lead to deeper drops. As mentioned, the profiles represent a moving average of the bending MOE over a length of $50 \mathrm{~mm}$. However, the pattern and trends of the profiles are not very sensitive to the distance over which the moving average is calculated, except that a shorter length would lead to deeper, and more narrow, drops of the profiles. Regarding the position of the weak sections along the board, there is very good agreement between the different curves, but different sets of data and different models indicate different loss of bending MOE due to the defects. Assuming that the profiles based on data from DIC represent the true 

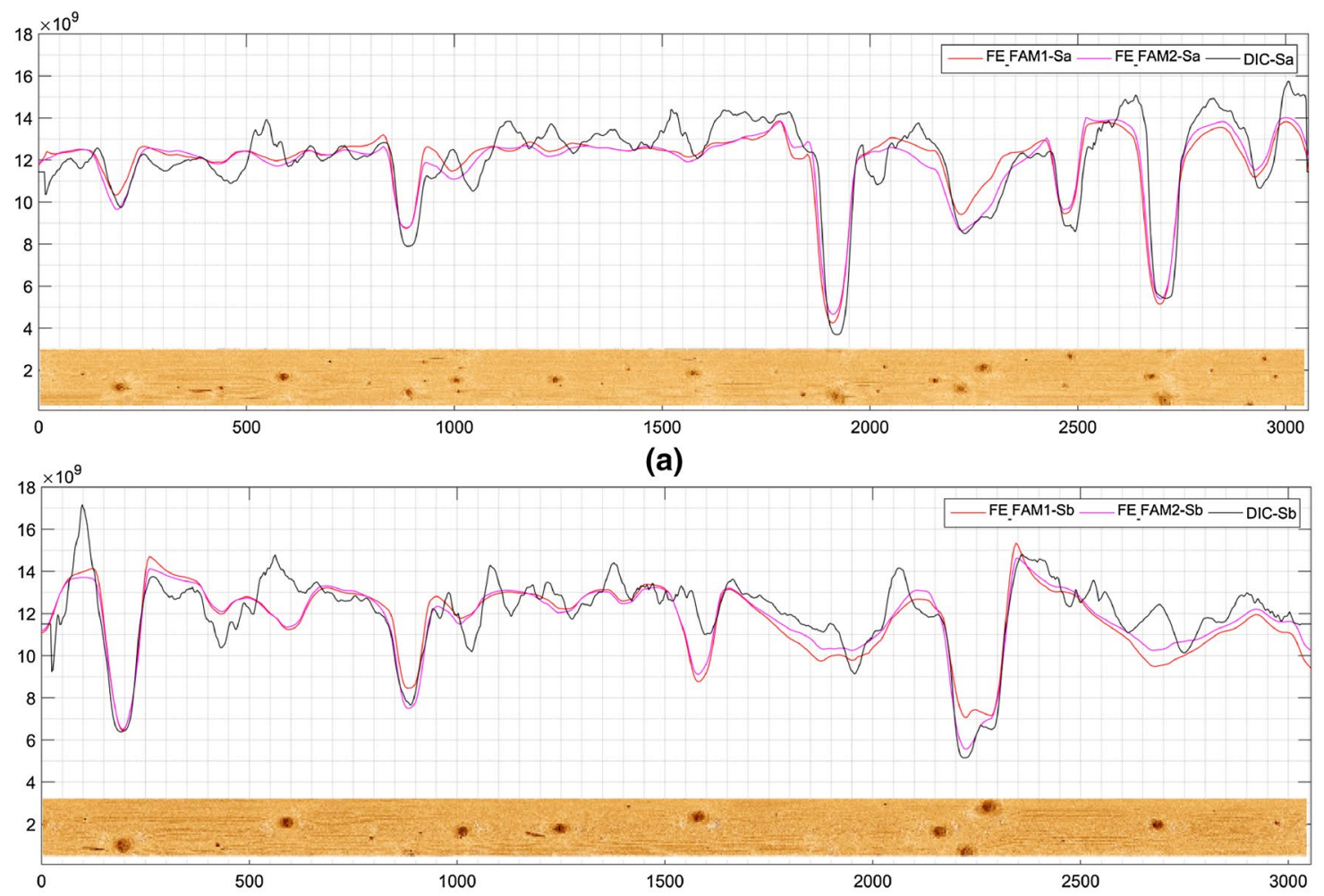

(b)

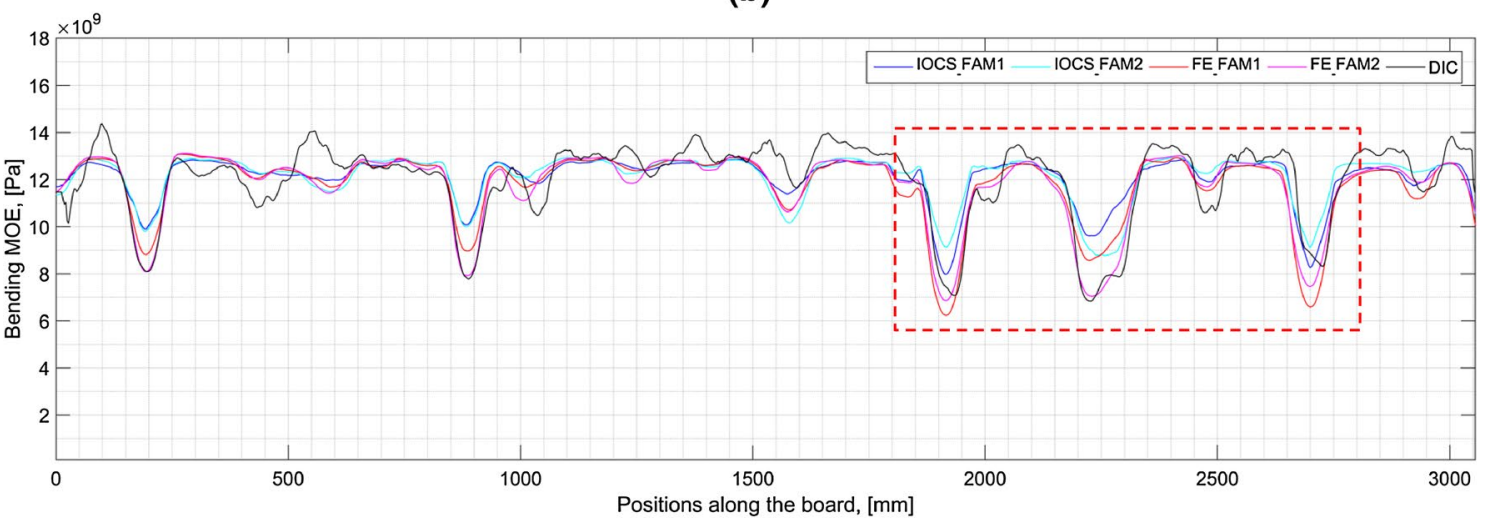

(c)

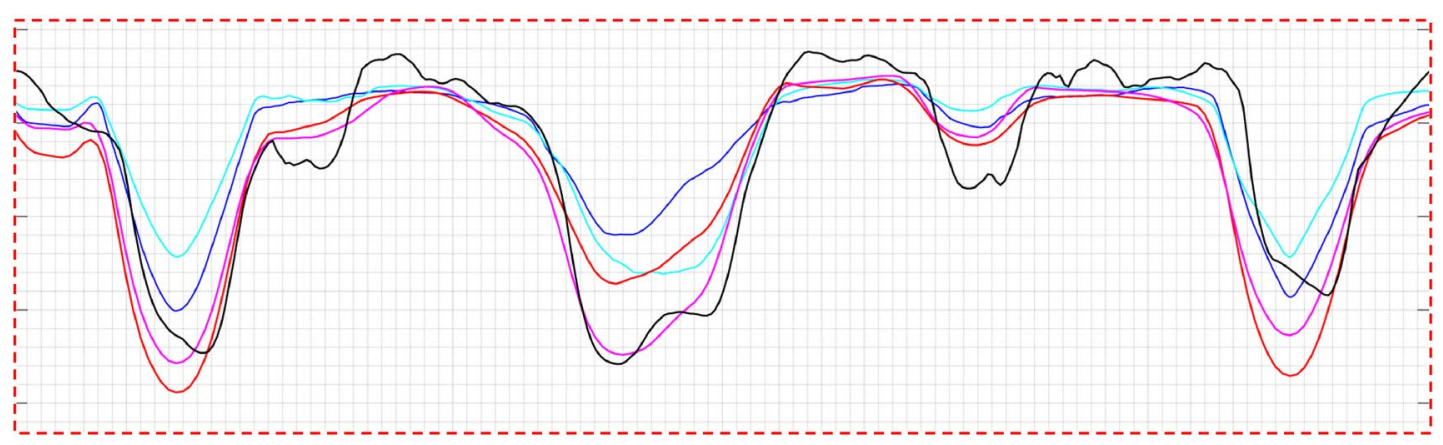

(d)

Fig. 9 Bending MOE profiles for specimen B2, each value of the profiles representing the average bending MOE over a surrounding distance of $50 \mathrm{~mm}$. a Bending MOE profiles calculated based on strains, of stress level corresponding to load stage 4 in Table 3, of surface $\mathrm{Sa}$ that originate from DIC, FE_FAM1 and FE_FAM2, respectively; b the corresponding bending MOE profiles based on strains of surface $\mathrm{Sb}$; $\mathbf{c}$ bending MOE profiles representing an average stiffness over the width of the board; $\mathbf{d}$ Enlarged view of the part of the board highlighted in $\mathbf{c}$ 
bending MOE variation along the board, comparisons of the curves of Fig. 9a, b show that FE_FAM2 give a more accurate representation of the bending MOE than what FE_ FAM1 does. When comparing the bending MOE profiles valid for the whole specimen shown in Fig. 9c, i.e. average bending MOE over the thickness of the board, and curves representing DIC, IOCS_FAM1, IOCS_FAM2, FE_FAM1 and FE_FAM2, respectively, the same conclusion can be drawn. In addition, it can be seen that the IOCS models overestimate the bending MOE at positions where knot clusters are located. This agrees with results presented by $\mathrm{Hu}$ et al. (2015). The FE models capture the reduction of bending MOE more accurately since more compliance, including shear deformations, were taken into account using these models. The MOE profile corresponding to the FE_FAM2 model complies very well with the profile obtained based on data from DIC.

\subsection{Values of material parameters for individual boards}

To validate the procedure for adjustment of the values of the material parameters for each board by multiplication of the scalar $\mu$, as described in Sect. 3.2, further investigations are needed. The current investigation, comprising only two boards, gave a rather comprehensive basis for evaluation of the ability of the models to capture variation in bending MOE along the boards since detailed MOE profiles of long boards are compared. However, the situation is different when assessing the procedure to determine board specific material parameters, since this procedure simply consists of multiplying nominal material parameters with a scalar. To get a sufficient basis to evaluate the procedure for adjustment of material parameters for individual boards, a complementary investigation comprising a higher number of boards, and in which one measure of global or local bending MOE is defined and used for comparison, should be performed.

\section{Conclusion}

A previously developed machine strength grading method, based on surface scanning of local fibre orientation and calculation of local bending stiffness, was the starting point of this work. Although the mentioned method gives rather accurate prediction of bending strength, it is based on simplifying assumptions when calculating local bending MOE. The purpose of the present paper was therefore to investigate the significance of these assumptions and the potential of calculating more accurate local bending MOE of boards.

DIC was used in laboratory tests to provide the basis for the calculation of strains on board surfaces and for the calculation of bending MOE profiles showing the actual compliance of timber subjected to pure bending. Then, two different models of fibre orientation within the volume of a board and two different mechanical models, of which the more advanced one was an elastic 3D FE model, were used to calculate strains and local bending MOE of the very same boards as those examined experimentally.

Strain fields obtained from FE modelling and simulation were in good agreement with those obtained by DIC. Strains calculated on the 3D FE model in combination with the herein suggested fibre angle model, gave a closer resemblance to strains from DIC than what the 3D FE model in combination with the simpler fibre angle model did.

A comparison of the performance of the two mechanical models showed that the 3D FE model was able to capture the compliance of boards containing knot clusters in a manner very similar to what was indicated by the results obtained from DIC, while the simpler IOCS model in many cases overestimated the local bending stiffness. When using the suggested fibre angle model, FAM2, in combination with the 3D FE model, the resemblance to the experimentally obtained profiles was very close, implying that the FE FAM2 model is sufficient to capture the variation in bending MOE along boards. For other purposes, i.e. when it is important that models of sawn timber accurately represent the radial and tangential material direction locally around knots, further improvements may still be necessary.

Bending MOE profiles provide the basis for IPs to bending strength and the herein suggested model should contribute to improved grading accuracy, since it seems to capture the variation in bending MOE related to fibre orientation far better than what previous models do. However, it is also crucial that board specific material parameters, i.e. MOEs and shear moduli, are determined accurately. Otherwise, calculated bending stiffness profiles may overestimate or underestimate the stiffness of the entire board, even if the relative variation in bending MOE is accurately represented. Thus, future research should address methods to determine material stiffness parameters of individual boards.

Open Access This article is distributed under the terms of the Creative Commons Attribution 4.0 International License (http://creativeco mmons.org/licenses/by/4.0/), which permits unrestricted use, distribution, and reproduction in any medium, provided you give appropriate credit to the original author(s) and the source, provide a link to the Creative Commons license, and indicate if changes were made.

\section{References}

Briggert A, Olsson A, Oscarsson J (2016a) Three-dimensional modelling of knots and pith location in Norway spruce boards using tracheid-effect scanning. Eur J Wood Prod 74(5):725-739

Briggert A, Hu M, Olsson A, Oscarsson J (2016b) Evaluation of three dimensional fibre orientation in Norway spruce using a laboratory 
laser scanner. In: Proceedings of World Conference on Timber Engineering. August 22-25. Vienna

Cramer SM, Goodman JR (1983) Model for stress analysis and strength prediction of lumber. Wood Fiber Sci 15(4):338-349

Dinwoodie JM (2000) Timber: its nature and behaviour. E \& FN Spon, New Fetter Lane

Foley C (2001) A three-dimensional paradigm of fibre orientation in timber. Wood Sci Technol 35(5):453-465

Foley C (2003) Modelling the effect of knots in structural timber. Report TVBK-1027. Dissertation, Lund University, Sweden

Goodman JR, Bodig J (1978) Mathematical model of the tension behavior of wood with knots and cross grain. In: Proceedings from the first International Conference on Wood Fracture. August 14-16. Banff, Alberta

Hackspiel C (2010) A numerical simulation tool for wood grading. Dissertation, Vienna University of Technology, Austria

Hankinson RL (1921) Investigation of crushing strength of spruce at varying angles of grain. Air Service Information Circular No. 259, US Air Service, USA

Hu M, Johansson M, Olsson A, Oscarsson J, Enquist B (2015) Local variation of modulus of elasticity in timber determined on the basis of non-contact deformation measurement and scanned fibre orientation. Eur J Wood Prod 73(1):17-27

Hu M, Olsson A, Johansson M, Oscarsson J, Serrano E (2016) Assessment of a three dimensional fiber orientation model for timber. Wood Fiber Sci 48(4):1-20

Hu M, Briggert A, Olsson A, Johansson M, Oscarsson J, Säll H (2018) Growth layer and fibre orientation around knots in Norway spruce: a laboratory investigation. Wood Sci Technol 52(1):7-27

Johansson C-J (2003) Grading of timber with respect to mechanical properties. In: Thelandersson S, Larsen HJ (eds) Timber engineering. Wiley, Chichester, pp 23-45

Johansson C-J, Boström L, Bräuner L, Hoffmeyer P, Holmquvist C, Solli KH (1998) Laminations for glued laminated timber-establishment of strength classes and for visual strength grade and machine settings for glulam laminations of Nordic origin. SP Swedish National Testing and Research Institute, SP Report, p 38

Kandler G, Lukacevic M, Füssl J (2016) An algorithm for the geometric reconstruction of knots within timber boards based on fibre angle measurements. Constr Build Mater 124:945-960

Lang R, Kaliske M (2013) Description of inhomogeneities in wooden structures: modelling of branches. Wood Sci Technol 47(5):1051-1070

Lukacevic M, Füssl J (2014) Numerical simulation tool for wooden boards with a physically based approach to identify structural failure. Eur J Wood Prod 72(4):497-508

Mattheck C (1997) Design in nature: learning from trees. Springer, Berlin, Heidelberg

Matthews PC, Beech BH (1976) Method and apparatus for detecting timber defects. US Patent no. 3976384, USA

Nystörm J (1999) Image based methods for nondestructive detection of compression wood in sawn timber. Licentiate thesis, Luleå University of Technology, Sweden
Nyström J (2003) Automatic measurement of fibre orientation in softwood by using the tracheid effect. Comput Electron Agric 41:91-99

Olsson A, Oscarsson J (2017) Strength grading on the basis of high resolution laser scanning and dynamic excitation: a full scale investigation of performance. Eur J Wood Prod 75(1):17-31

Olsson A, Oscarsson J, Serrano E, Källsner B, Johansson M, Enquist B (2013) Prediction of timber bending strength and in-member cross-sectional stiffness variation on the basis of local wood fibre orientation. Eur J Wood Prod 71(3):319-333

Ormarsson S (1999) Numerical analysis of moisture-related distortions in sawn timber. Publication 99:7. Dissertation, Chalmers University of Technology, Sweden

Oscarsson J, Olsson A, Enquist B (2012) Strain fields around knots in Norway spruce specimens exposed to tensile forces. Wood Sci Technol 46(4):593-610

Oscarsson J, Olsson A, Enquist B (2014) Localized modulus of elasticity in timber and its significance for the accuracy of machine strength grading. Wood Fiber Sci 46(4):489-501

Petersson H (2010) Use of optical and laser scanning techniques as tools for obtaining improved FE-input data for strength and shape stability analysis of wood and timber. In: Proceedings of the IV European conference on computational mechanics, May 16-21. Paris

Seltman J (1992) Indication of slope-of-grain and biodegradation in wood with electromagnetic waves. In: Lindgren O (ed) First international seminar on scanning technology and image processing on wood. Luleå University of Technology, Skellefteå

Simonaho S-P, Palviainen J, Tolonen Y, Silvennoinen R (2004) Determination of wood grain direction from laser light scattering pattern. Opt Laser Eng 41:95-103

Sjödin J, Serrano E, Enquist B (2006) Contact-free measurements and numerical analysis of the strain distribution in the joint area of steel-to-timber dowel joints. Holz Roh Werkst 64(6):497-506

Soest J, Matthews PC, Wilson B (1993) A simple optical scanner for grain defects. In: Proceedings of 5th International Conference on Scanning Technology and Process Control for the Wood Products Industry, October 25-27. Atlanta

Viguier J, Bourreau D, Bocquet J-F, Pot G, Bléron L, Lanvin J-D (2017) Modelling mechanical properties of spruce and Douglas fir timber by means of X-ray and grain angle measurements for strength grading purpose. Eur J Wood Prod 75(4):527-541

WoodEye AB (2017) WoodEye. http://woodeye.se/en/. Accessed 4 October 2017

Publisher's Note Springer Nature remains neutral with regard to jurisdictional claims in published maps and institutional affiliations. 